\title{
Asymptotic behaviour of monotone multi-valued dynamical systems
}

\author{
T. Caraballo ${ }^{1}$, J.A. Langa ${ }^{1}$ And J. VAlero ${ }^{2}$ \\ ${ }^{1}$ Departamento de Ecuaciones Diferenciales Análisis Numérico, \\ Universidad de Sevilla, Apdo. de Correos 1160, 41080-Sevilla, Spain. \\ e-mails: caraball@us.es; langa@us.es \\ ${ }^{2}$ Centro de Investigación Operativa \\ Universidad Miguel Hernández, Avda. Universidad, s/n. - Elche - 03202 \\ e-mail: jvalero@umh.es
}

\begin{abstract}
We introduce the concept of a monotone multi-valued semi-flow as an order-preserving map. This definition is motivated by the applications in the theory of differential equations without uniqueness of solutions. For an order preserving multi-valued semi-flow we prove several results on the structure of the global attractor. Some applications to models governed by ordinary differential equations and delay equations with continuous right-hand side are presented. In particular, the abstract results are applied to a biochemical control circuit.
\end{abstract}

Key words: Monotone dynamical systems, order-preserving semi-flows, multi-valued systems, global attractor, ordinary differential equations, equations with delay

AMS Subject Classification (2000): 34C12, 34D05, 34D23, 34D45, 34K12

\section{Introduction}

There exists a wide literature on the theory of monotone methods and comparison principles for differential equations; that is, ordered initial states remain ordered for subsequent times (among many others, see, for example, the monographs of Smith [20] or Hirsch [13]). Actually, this theory has become an important branch in the theory of dynamical systems. In particular, monotonicity leads to some important properties of the asymptotic behaviour of the models; among them, the forward evolution of intervals, the existence of fixed points for monotone dynamical systems, and some properties related to the structure of attracting sets.

But none of these concepts is well defined when we have a multi-valued dynamical system related, for example, to a differential equation without uniqueness of solutions, or to a differential inclusion. In these cases, the concept of an order, and even those of sub, super-equilibria, and equilibria becomes unclear. In this paper we contribute to the theory of order-preserving multi-valued dynamical systems, by proposing a suitable definition of an order-preserving multi-valued semi-flow $G$, which has a direct application to a variety of models. If we suppose that the multi-valued dynamical system has a global attractor in the sense of Melnik and Valero [17], we then prove our main theorem, which gives the existence of maximal and minimal equilibria on the attractor in which all the asymptotic behaviour of the system is then confined, providing also some information on the stability properties of these equilibria and on the structure of the global attractor. In particular, under some conditions we show the existence of two equilibria which attract all the bounded sets allocated, respectively, above or below these values. To our knowledge, this is the first time in which the well developed techniques related to monotone dynamical systems (see, for example, Smith [20]) have been applied to analyse the asymptotic behaviour of multi-valued semi-flows. 
Some applications to differential equations without uniqueness of solutions are developed in the following section. In all of them, we prove that $G$ is order preserving, so that our main theorem can be applied. Also, under some conditions we prove that the set $G\left(t, x_{0}\right)$, corresponding to a fixed initial data and a fixed $t$, is a totally ordered set. The results are applied to cooperative systems of differential equations and to scalar delay equations. In particular, we consider a model of a biochemical control circuit.

Finally, we note that these results could be extended to other situations, as differential inclusions, or non-autonomous and stochastic differential equations and inclusions (see [1], [10], where monotone systems are studied for stochastic equations with uniqueness of solutions). This is part of our future work.

\section{Monotone multi-valued dynamical systems}

Let $(X, \rho)$ be a complete metric space and let $P(X)$ be the set of all non-empty subsets of $X$. Denote

$$
\begin{aligned}
\mathcal{B}(X) & =\{A \in P(X): A \text { is bounded }\}, \\
C(X) & =\{A \in P(X): A \text { is closed }\}, \\
K(X) & =\{A \in P(X): A \text { is compact }\} \\
\operatorname{dist}(A, B) & =\sup _{x \in A} \inf _{y \in B} \rho(x, y), \text { for } A, B \subset X .
\end{aligned}
$$

Definition 1 A map $G: \mathbb{R}_{+} \times X \rightarrow P(X)$ is said to be a multi-valued semi-flow (MSF) on $X$ if:

i) $G(0, \cdot)=I d$, i.e., the identity map;

ii) $G(t+s, x) \subset G(t, G(s, x))$, for all $x \in X, s, t \in \mathbb{R}_{+}$.

It is called a strict multi-valued semi-flow (SMSF) if, moreover, $G(t+s, x)=G(t, G(s, x))$.

Multi-valued semi-flows are a natural generalization of the semigroups of operators for equations without uniqueness (see [4], [15], [14], [17], [18], [19], [22]). However, other approaches, such as generalized semigroups ([5], [6], [11]), have also been used (see [8] for a comparison of the two methods with respect to the theory of attractors).

Order preserving semigroups have been used widely and fruitfully in the literature. Among many other applications, this property has enabled the proof of some nice properties on the asymptotic behaviour of solutions for ordinary and partial differential equations (see [1], [2], [10], [20]). The question about the possible generalization of this rich concept to the multi-valued case arises naturally. In the following subsections we give such a generalization concerning the asymptotic behaviour of these systems.

\subsection{Order preserving multi-valued semi-flows}

We now introduce the concept of order-preserving (or monotone) multi-valued systems.

Definition $2 A M S F\{G(t, \cdot): X \rightarrow C(X)\}$ is said to be order-preserving if there exists an order relation ' $\leq$ ' in $X$ such that, if $x_{0} \leq y_{0}$, then $G\left(t, x_{0}\right) \leq G\left(t, y_{0}\right)$, for all $t \geq 0$, in the sense that

a) There exists $\underline{x}(t) \in G\left(t, x_{0}\right)$ such that

$$
\underline{x}(t) \leq y(t), \quad \text { for all } y(t) \in G\left(t, y_{0}\right) ;
$$

b) There exists $\bar{y}(t) \in G\left(t, y_{0}\right)$ such that

$$
x(t) \leq \bar{y}(t), \quad \text { for all } x(t) \in G\left(t, x_{0}\right) .
$$

In particular, it follows from the definition the existence of maximal and minimal elements in $G\left(t, x_{0}\right)$, so that $\underline{x}(t)($ resp. $\bar{y}(t))$ can be chosen independent of $y_{0}$ (resp. $\left.x_{0}\right)$ if $y_{0} \neq x_{0}$.

It is important to assume that the order is compatible with the topology in the following sense: 
1. Any bounded subset $B$ of $X$ is contained in some "interval", i.e., there exist $a \leq b$ such that $B \subset$ $[a, b]=\{x \in X: a \leq x \leq b\}$.

2. If $x_{n} \rightarrow x, y_{n} \rightarrow y$ and $x_{n} \leq y_{n}$, then $x \leq y$.

The second condition implies that any interval $[a, b]$ is closed.

\subsection{Global attractors for order-preserving multi-valued semi-flows}

Now we briefly recall some concepts and results from the theory of attractors for MSF.

Definition $3 A$ set $\mathcal{A} \subset X$ is said to be a global attractor associated to $G$ if:

i) $\mathcal{A} \subset G(t, \mathcal{A})$, for all $t \in \mathbb{R}_{+}$, that is, it is negatively semi-invariant;

ii) $\mathcal{A}$ attracts any bounded subset $D \subset X$, i.e.

$$
\lim _{t \rightarrow+\infty} \operatorname{dist}(G(t, D), \mathcal{A})=0 .
$$

In applications it is desirable to prove also that $\mathcal{A}$ is compact and strictly invariant, i.e.

$$
\mathcal{A}=G(t, \mathcal{A}), \text { for all } t \in \mathbb{R}_{+} .
$$

We now establish some sufficient conditions that guarantee the existence of a global attractor (see [17]). Suppose the following conditions for the MSF $G$ :

(H1) $G$ is point dissipative, i.e., there exists a bounded subset $B_{0}$ such that for every $x \in X$ there is $t_{x}$ for which

$$
G(t, x) \subset B_{0} \text {, for all } t \geq t_{x} .
$$

(H2) The set $\gamma_{0}^{+}(B)=\cup_{t \geq 0} G(t, B)$ is bounded for every $B \in \mathcal{B}(X)$ and $G$ is asymptotically compact, i.e., any sequence $\xi_{n} \in G\left(t_{n}, B\right)$, where $t_{n} \rightarrow+\infty$, is pre-compact for all $B \in \mathcal{B}(X)$.

(H3) $G(t, \cdot): X \rightarrow C(X)$ is upper semi-continuous, that is, for all $t \in \mathbb{R}_{+}$, given $x \in X$ and a neighbourhood $\mathcal{O}(G(t, x))$ of $G(t, x)$, there exists $\delta>0$ such that if $\rho(x, y)<\delta$ then

$$
G(t, y) \subset \mathcal{O}(G(t, x)) .
$$

The following theorem is a direct consequence of Lemma 1, Theorem 3 and Remark 8 in [17]:

Theorem 4 If $(H 1)-(H 3)$ hold, then $G$ has a global compact attractor $\mathcal{A}$. If, in addition, $G$ is a strict semi-flow, then $\mathcal{A}$ is strictly invariant.

Definition 5 A point $x \in X$ is said to be an equilibrium (or a fixed point) of $G$ if

$$
x \in G(t, x), \text { for all } t \geq 0 .
$$

The following result provides sufficient conditions for the existence of upper and lower asymptotically stable equilibria, as well as some information on the structure of the global attractor.

Theorem 6 Let $G$ be an order-preserving SMSF satisfying $(H 1)-(H 3)$ and $\mathcal{A}$ its associated global compact invariant attractor. Then, there exist equilibria $x_{*}, y^{*} \in \mathcal{A}$ such that:

1. $x_{*} \leq y^{*}$ and

$$
\mathcal{A} \subseteq\left[x_{*}, y^{*}\right] .
$$


2. $x_{*}$ (resp. $\left.y^{*}\right)$ is minimal (resp. maximal) in the sense that any other fixed points are contained in the interval $\left[x_{*}, y^{*}\right]$.

If the solutions corresponding to the initial conditions $x_{*}$ and $y^{*}$ are unique (i.e. if $G\left(t, x_{*}\right)=x_{*}$ and $G\left(t, y^{*}\right)=y^{*}$ for all $\left.t \in \mathbb{R}\right)$, then:

3. $x_{*}$ is globally attracting from below, that is, for all $v \in X$ with $v \leq x_{*}$, we have that

$$
\lim _{t \rightarrow+\infty} \operatorname{dist}\left(G(t, v), x_{*}\right)=0 .
$$

4. $y^{*}$ is globally attracting from above, that is, for all $v \in X$ with $y^{*} \leq v$ we have that

$$
\lim _{t \rightarrow+\infty} \operatorname{dist}\left(G(t, v), y^{*}\right)=0 .
$$

Proof. We note that $\mathcal{A}$ is bounded, and then by the condition of compatibility of the order with the topology there exist $\underline{x} \leq \bar{y}$ such that $\mathcal{A} \subseteq[\underline{x}, \bar{y}]$. Hence,

$$
\underline{x} \leq u \leq \bar{y}, \text { for all } u \in \mathcal{A}
$$

Since $G$ is order preserving, we have

$$
G(t, \underline{x}) \leq G(t, u) \leq G(t, \bar{y}),
$$

so that, there exist $\underline{x}(t, u) \in G(t, \underline{x})$ and $\bar{y}(t, u) \in G(t, \bar{y})$ such that

$$
\underline{x}(t, u) \leq z \leq \bar{y}(t, u) \text {, for all } z \in G(t, u), u \in \mathcal{A} .
$$

But note also that $G(t, x) \leq G(t, x)$ implies that $G(t, x)$ has a minimal and a maximal element, and then there exist $\underline{x}(t) \in G(t, \underline{x})$ and $\bar{y}(t) \in G(t, \bar{y})$ (not depending on $u$ ) such that

$$
\underline{x}(t) \leq z \leq \bar{y}(t), \text { for all } z \in G(t, u), u \in \mathcal{A} .
$$

Thus, since $\mathcal{A} \subset G(t, \mathcal{A})$ we have

$$
\underline{x}(t) \leq u \leq \bar{y}(t) \text {, for all } u \in \mathcal{A} .
$$

The compact set $\mathcal{A}$ attracts $\underline{x}$ and $\bar{y}$, so that $\operatorname{dist}(\underline{x}(t), \mathcal{A}) \rightarrow 0$, $\operatorname{dist}(\bar{y}(t), \mathcal{A}) \rightarrow 0$, as $t \rightarrow+\infty$, and then we can choose converging subsequences $\underline{x}\left(t_{n}\right) \rightarrow x_{*} \in \mathcal{A}, \bar{y}\left(t_{n}\right) \rightarrow y^{*} \in \mathcal{A}$. The compatibility of the order with the topology gives

$$
x_{*} \leq u \leq y^{*}, \text { for all } u \in \mathcal{A} .
$$

In order to check that $x_{*}, y^{*}$ are equilibria we first note that $x_{*}, y^{*} \in \mathcal{A}$ implies that these points are uniquely determined by (2). Indeed, if $x_{*}^{1}, x_{*}^{2} \in \mathcal{A}$ are two points satisfying (2), then $x_{*}^{1} \leq x_{*}^{2}$ and $x_{*}^{1} \geq x_{*}^{2}$, so that $x_{*}^{1}=x_{*}^{2}$. Since the attractor $\mathcal{A}$ is negatively semi-invariant and the MSF $G$ order preserving, for any $t \geq 0$ there exist $\underline{x}(t) \in G\left(t, x_{*}\right), \bar{y}(t) \in G\left(t, y^{*}\right)$ such that

$$
\underline{x}(t) \leq u \leq \bar{y}(t) \text {, for all } u \in \mathcal{A} .
$$

But $\mathcal{A}$ is also positively semi-invariant, so that $\underline{x}(t), \bar{y}(t) \in \mathcal{A}$, and then $\underline{x}(t)=x_{*}, \bar{y}(t)=y^{*}$.

It is clear that any equilibrium has to be contained in the global attractor (by the attractivity property), so that point 2 of the theorem is also proved.

Finally, if the solution corresponding to $x_{*}$ is unique, then for any $v \leq x_{*}$ we have

$$
\begin{gathered}
G(t, v) \leq G\left(t, x_{*}\right)=x_{*}, \\
\operatorname{dist}(G(t, v), \mathcal{A}) \rightarrow 0, \text { as } t \rightarrow+\infty,
\end{gathered}
$$

so that the only possibility is $\operatorname{dist}\left(G(t, v), x_{*}\right) \rightarrow 0$. The same argument is valid for $y^{*}$. 


\section{Applications}

In this section we apply our theory to some systems modeled by ordinary differential equations and delay equations without uniqueness of solutions, so that a multi-valued semi-flow has to be defined. Under some conditions, we show that the corresponding semi-flow is monotone, so that our previous results can be applied.

\subsection{Ordinary differential equations with continuous right hand-side}

\subsubsection{An $N$-dimensional case: a cooperative system}

Consider the usual order in $\mathbb{R}^{N}$, i.e.

$$
x \leq y \Longleftrightarrow x_{i} \leq y_{i} \text { for all } i=1,2, \ldots, N .
$$

We say that $x<y$ if $x \leq y$ and there exists $j \in\{1,2, \ldots, N\}$ such that $x_{j}<y_{j}$.

Let us now consider the system

$$
\left\{\begin{array}{l}
x^{\prime}(t)=f(x(t)) \\
x(0)=x_{0}
\end{array}\right.
$$

with $f: \mathbb{R}^{N} \longrightarrow \mathbb{R}^{N}$ continuous and satisfying

$$
(f(x), x) \leq c_{1}\|x\|^{2}+c_{2}, c_{1} \in \mathbb{R}, c_{2}>0,
$$

where $(\cdot, \cdot),\|\cdot\|$ are respectively the scalar product and the norm in $\mathbb{R}^{N}$. Suppose also the following monotonicity hypothesis on $f$ :

$$
f_{i}(x) \leq f_{i}(y), \quad \text { if } x, y \in \mathbb{R}^{N}, x_{i}=y_{i} \text {, and } x \leq y .
$$

Condition (4) implies easily that any solution is globally defined in time, and for any $T>0$ the set of solutions is uniformly bounded in bounded sets of initial data, so that, for all $B \subset \mathbb{R}^{N}$ bounded and $T>0$, there exists $C(B, T)$ such that $\sup _{t \in[0, T]}|x(t)| \leq C(B, T)$, where $x(t)$ denotes any solution of (3) corresponding to $x_{0} \in B$. Denote by $S\left(x_{0}\right) \subset C\left([0,+\infty), \mathbb{R}_{+}^{N}\right)$ the set of all solutions with initial condition $x_{0}$. Define also the set

$$
S\left(x_{0}, T\right)=\left\{x(\cdot) \in C\left([0, T], \mathbb{R}^{N}\right): x(\cdot) \text { is a solution of }(3) \text { and } x(0)=x_{0}\right\},
$$

which is compact in $C\left([0, T], \mathbb{R}^{N}\right)$ by the Ascoli-Arzelà theorem, and the map $G: \mathbb{R}_{+} \times \mathbb{R}^{N} \longrightarrow K\left(\mathbb{R}^{N}\right)$ by

$$
G\left(t, x_{0}\right)=\left\{x(t): x(\cdot) \in S\left(x_{0}\right)\right\} .
$$

It is easy to check that $G$ is a strict multi-valued semi-flow. The inclusion $G(t+s, x) \subset G(t, G(s, x))$ follows from the fact that if $x(\cdot) \in S\left(x_{0}, t+s\right)$, then $y(\cdot)=x(s+\cdot) \in S(x(s), t)$. The converse inclusion $G(t, G(s, x)) \subset G(t+s, x)$ follows from the concatenation property: if $x(\cdot) \in S\left(x_{0}, s\right)$ and $y(\cdot) \in$ $S(x(s), t)$, then

$$
z(r)=\left\{\begin{aligned}
x(r), & \text { if } 0 \leq r \leq s, \\
y(r-s), & \text { if } s \leq r \leq s+t,
\end{aligned}\right.
$$

belongs to $S\left(x_{0}, t+s\right)$. Hence, we have the SMSF $G: \mathbb{R}_{+} \times \mathbb{R}^{N} \longrightarrow K\left(\mathbb{R}^{N}\right)$.

We also note that the set of fixed points coincides with the set of zeros of $f$. Indeed, if $x_{0}$ is a fixed point and for some $i$, say, $f_{i}\left(x_{0}\right)>0$, then for any $x(\cdot) \in S\left(x_{0}\right)$ we have $x_{i}^{\prime}(t)>0$ for $t \in[0, \delta]$, so that $x_{i}(t)>x_{0 i}$ in $(0, \delta]$, and $x_{0} \notin G\left(t, x_{0}\right)$. Hence, $x_{0}$ is not an equilibrium. Conversely, if $f\left(x_{0}\right)=0$, then $x(t)=x_{0}$, for all $t$, is a solution and then $x_{0} \in G\left(t, x_{0}\right)$.

In many physical and biological applications the variables $x_{i}$ have to be non-negative. Hence, we need to define a multi-valued semi-flow in the phase space $\mathbb{R}_{+}^{N}$ instead of $\mathbb{R}^{N}$. 
Lemma 7 Suppose that

$$
f_{i}(x) \geq 0, \text { for all } i \text { and } x \in \mathbb{R}_{+}^{N} \text { such that } x_{i}=0 .
$$

Then, for any $x_{0} \geq 0$ (i.e. $x_{0 i} \geq 0$ for all $i$ ) there exists at least one global solution such that $x(t) \geq 0$, for any $t \geq 0$.

Proof. Define the approximate functions $f^{\varepsilon}(x)=f(x)+\varepsilon d, \varepsilon>0$, where $d=(1, \ldots, 1)$, which satisfies $f_{i}^{\varepsilon}(x) \geq \varepsilon$, for all $i$ and $x \in \mathbb{R}_{+}^{N}$ such that $x_{i}=0$. Consider an arbitrary solution $x^{\varepsilon}(t)$ of the equation $x^{\prime}=f^{\varepsilon}(x)$ corresponding to $x_{0} \geq 0$. Suppose $x^{\varepsilon}(t) \geq 0$, for some $t$. Let the $i$ component of this solution be negative in some interval $\left(t_{0}, t_{1}\right), x_{i}(t) \geq 0$, for $t \leq t_{0}$, with $i$ the first component which becomes negative. Thus, by the continuity of $f^{\varepsilon}$ we have

$$
\frac{d}{d t} x_{i}^{\varepsilon}(t)=f_{i}^{\varepsilon}\left(x^{\varepsilon}(t)\right)>0, \text { for } t \in\left(t_{0}, t_{0}+\delta\right),
$$

which is a contradiction. Hence, $x^{\varepsilon}(t) \geq 0$, for all $t \geq 0$.

Fix $T>0$. Then by (4) and the continuity of $f$ the functions $x^{\varepsilon}$ and $\frac{d}{d t} x^{\varepsilon}$ are uniformly bounded in $C\left([0, T], \mathbb{R}^{N}\right)$, so that by the Ascoli-Arzelá theorem there exists a converging subsequence $x^{\varepsilon_{n}}$. The limit $x(\cdot)$ is a solution of $(3)$ in $[0, T]$ and $x(t) \geq 0$, for all $t \in[0, T]$, as can be easily checked. Repeating the same in the intervals $[T, 2 T],[2 T, 3 T]$, etc., and using a diagonal argument we obtain a globally defined solution $x(\cdot)$ such that $x(t) \geq 0$, for $t \geq 0$, as desired.

Remark 8 This lemma does not guarantee that all the solutions are non-negative, as happens in the case of equations with uniqueness. This can be checked in the following simple example:

$$
x^{\prime}=\operatorname{sign}(x) \sqrt{|x|}, x(0)=0,
$$

where $\operatorname{sign}(x)=1$ (resp. $-1,0)$, if $x>0$ (resp. $<0,=0)$, We have the solutions $x_{1}(t)=0, x_{2}(t)=\frac{t^{2}}{4}$, $x_{3}(t)=-\frac{t^{2}}{4}$ among many others.

Denote by $U\left(x_{0}\right) \subset C\left([0,+\infty), \mathbb{R}_{+}^{N}\right)$ the set of all solutions with initial condition $x_{0}$ such that $x(t) \geq 0$ for all $t \geq 0$. Define also the set

$$
D\left(x_{0}, T\right)=\left\{x(\cdot) \in C\left([0, T], \mathbb{R}^{N}\right): x(\cdot) \text { is a solution of }(3) \text { and } x(0)=x_{0}, x(t) \geq 0\right\}
$$

and the map $U: \mathbb{R}_{+} \times \mathbb{R}_{+}^{N} \longrightarrow K\left(\mathbb{R}_{+}^{N}\right)$ :

$$
U(t, x)=\left\{x(t): x(\cdot) \in D\left(x_{0}\right)\right\} .
$$

$U$ is a SMSF. We note that $D\left(x_{0}, T\right)$ is a closed subset of $S\left(x_{0}, T\right)$, so that it is compact. Therefore, $U\left(t, x_{0}\right)$ is compact as well.

We are going to study whether the SMSF $G$ and $U$ are order preserving. Let us begin with the map $G$.

Lemma 9 Let conditions (4) and (5) hold. Then for any $x_{0} \leq y_{0}$ there exists two solutions $\bar{y}(\cdot) \in S\left(T, y_{0}\right)$, $\underline{x}(\cdot) \in S\left(T, x_{0}\right)$, such that

$$
\begin{aligned}
& \bar{y}(t) \geq x(t), \text { for all } t \in[0, T] \text { and } x(\cdot) \in S\left(T, x_{0}\right), \\
& \underline{x}(t) \leq y(t), \text { for all } t \in[0, T] \text { and } y(\cdot) \in S\left(T, y_{0}\right) .
\end{aligned}
$$

Hence, $G$ is order preserving. 
Proof. Define the functions $g^{\varepsilon}(x)=f(x)+\varepsilon d, \varepsilon>0$, where $d=(1, \ldots, 1)$. It is clear from (5) that

$$
g_{i}^{\varepsilon}(y) \geq f_{i}(x)+\varepsilon, \text { if } x_{i}=y_{i} \text { and } x \leq y .
$$

Let $y_{\varepsilon}(\cdot), y_{\varepsilon}(0)=y_{0}$, be a solution of (3) where $f$ is replaced by $g_{\varepsilon}$, and let $x(\cdot) \in S\left(T, x_{0}\right)$ be arbitrary. We claim that $y_{\varepsilon}(t) \geq x(t)$, for all $t \in[0, T]$. If not, then there exist a time $0 \leq t_{0}<T$ and $i, \delta>0$ such that

$$
\begin{gathered}
x_{i}\left(t_{0}\right)=y_{i}\left(t_{0}\right), y\left(t_{0}\right) \geq x\left(t_{0}\right), \\
y_{\varepsilon i}(t)<x_{i}(t), \text { for all } t_{0}<t \leq t_{0}+\delta .
\end{gathered}
$$

Then (7) gives $g_{i}^{\varepsilon}\left(y\left(t_{0}\right)\right) \geq \varepsilon+f_{i}\left(x\left(t_{0}\right)\right)$ and the continuity of the functions $g_{\varepsilon}, f$ implies that

$$
\frac{d}{d t}\left(y_{\varepsilon i}(t)-x_{i}(t)\right)=g_{i}^{\varepsilon}(y(t))-f_{i}(x(t))>0, \text { for all } t \in\left(t_{0}, t_{0}+\tilde{\delta}\right),
$$

for some $\tilde{\delta} \leq \delta$, and thus $y_{\varepsilon i}(t)>x_{i}(t)$, which is a contradiction.

Further, by (4) and the continuity of $f$ we obtain that the functions $y_{\varepsilon}, \frac{d}{d t} y_{\varepsilon}$ are uniformly bounded in $C\left([0, T], \mathbb{R}^{N}\right)$, so that by the Ascoli-Arzelà theorem there exists a converging subsequence $y_{\varepsilon_{n}}$. The limit $\bar{y}(t)$ is a solution of $(3)$ and clearly $\bar{y}(t) \geq x(t)$, for all $t \in[0, T]$.

The existence of the function $\underline{x}(\cdot)$ can be proved in a similar way by defining the function $g^{\varepsilon}(x)=$ $f(x)-\varepsilon d$.

This means that $G$ is order preserving.

We have the following result:

Theorem 10 Let conditions (4) and (5) hold. Assume also that (H1)-(H2) are satisfied. Then G satisfies also (H3) and the statement of Theorem 6 holds true.

Remark 11 In this case $(H 2)$ is equivalent to the fact that the set $\gamma_{0}^{+}(B)$ is bounded for any $B$ bounded.

Remark 12 In the scalar case, that is $N=1$, the order preserving property is satisfied without assuming any condition of the type (5) [12, p.27, Corollary 4.2].

Proof. We have already seen that $G(t, x)$ is a compact set, so that in order to get (H3) it remains to prove that the map $G(t, \cdot)$ is upper semi-continuous. Arguing again by contradiction, take a sequence $y_{n} \in G\left(t, x_{n}\right), x_{n} \rightarrow x_{0}$, and a neighborhood $\mathcal{N}$ of $G\left(t, x_{0}\right)$, such that $y_{n} \notin \mathcal{N}$. If $x_{n}(\cdot) \in S\left(x_{n}, t\right)$ are such that $x_{n}(0)=x_{n}, y_{n}=x_{n}(t)$, then, by $(4)$, we have that $x_{n}(t)$ and $x_{n}^{\prime}(t)$ are uniformly bounded in $[0, t]$. The Ascoli-Arzelà theorem gives (up to a subsequence) $x_{n}(\cdot) \rightarrow x(\cdot)$ in $C\left([0, t], \mathbb{R}^{N}\right)$. Hence,

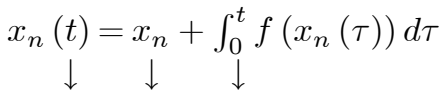

$$
\begin{aligned}
& x(t)=x_{0}+\int_{0}^{t} f(x(\tau)) d \tau .
\end{aligned}
$$

Therefore, $x(\cdot) \in S\left(x_{0}, t\right)$ and $x(t) \in G\left(t, x_{0}\right)$, which is a contradiction. (H3) is then proved.

Since $G$ is order preserving by Lemma 9 , we can apply Theorem 6 .

Consider now the map $U$. We note first that if we try to repeat the same proof of Lemma 6 , then one can get the existence of the solution $\bar{y}(\cdot)$. However, the proof for the solution $\underline{x}(\cdot)$ fails because, when we take the function $g^{\varepsilon}(x)=f(x)-\varepsilon d$, the solutions of (3) are not in general non-negative. We will be able to prove the order preserving property for $U$ under more restrictive conditions.

We shall use the following condition:

$$
f_{i}(x)<f_{i}(y), \quad \text { if } x, y \in \mathbb{R}^{N}, x_{i}=y_{i} \text {, and } x<y .
$$


Theorem 13 Let conditions (4), (6) and (8) hold and let the set $U(t, x)$ have a minimal and a maximal element for any $(t, x)$. Then $U$ is order preserving.

If we assume that $(H 1)-(H 2)$ are satisfied, then $(H 3)$ also holds and the statement of Theorem 6 holds true.

Proof. Denote by $\bar{x}$ and $\underline{x}$ the maximal and the minimal element in $U\left(T, x_{0}\right)$, and let $\bar{x}(t)$ be a solution corresponding to the maximal element $\bar{x} \in U\left(T, x_{0}\right)$.

Take $y_{0}<x_{0}$ and $y(\cdot) \in D\left(T, y_{0}\right)$. If $y\left(t_{0}\right)=\bar{x}\left(t_{0}\right)$ for some $t_{0}$, then we define $x(\cdot) \in D\left(x_{0}, T\right)$ as

$$
x(t)= \begin{cases}\bar{x}(t), & \text { if } 0 \leq t \leq t_{0}, \\ y(t), & \text { if } t_{0} \leq t \leq T .\end{cases}
$$

Since $x(T) \in U\left(T, x_{0}\right)$ and $\bar{x}(T)$ is the maximal element of $U\left(T, x_{0}\right)$, we have $x(T)=y(T) \leq \bar{x}(T)=\bar{x}$. Assume then that $y(T) \leq \bar{x}$ is not true and $y\left(t_{0}\right) \neq \bar{x}\left(t_{0}\right)$ for all $t_{0}$. Then there exists $i \in\{1, \ldots, N\}, t_{0}<T$ and $\delta>0$ such that

$$
\begin{gathered}
\bar{x}_{i}\left(t_{0}\right)=y_{i}\left(t_{0}\right), x\left(t_{0}\right) \neq y\left(t_{0}\right), y\left(t_{0}\right) \leq x\left(t_{0}\right), \\
y_{i}(t)>\bar{x}_{i}(t) \text { for all } t_{0}<t \leq t_{0}+\delta .
\end{gathered}
$$

Thus, by (8), we have that $f_{i}\left(y\left(t_{0}\right)\right)-f_{i}\left(\bar{x}\left(t_{0}\right)\right)<0$. Then, the continuity of $f$ implies that

$$
\frac{d}{d t}\left(y_{i}(t)-\bar{x}_{i}(t)\right)=f_{i}(y(t))-f_{i}(\bar{x}(t))<0, \text { for all } t \in\left(t_{0}, t_{0}+\tilde{\delta}\right),
$$

for some $\tilde{\delta} \leq \delta$, and thus $y_{i}(t)-\bar{x}_{i}(t)<0$, which is a contradiction.

Therefore, repeating a similar argument for $\underline{x}$, it follows that $U$ is order preserving.

We conclude by repeating the same proof of Theorem 10 .

In order to prove that the set $U(T, x)$ always has maximal and minimal points we need to assume an additional condition on the function $f$.

Let us now consider the case $N=2$ and assume the additional condition:

$$
f_{i}(x)<f_{i}(y) \text {, if } x, y \in \mathbb{R}^{2}, x_{i} \geq y_{i}, x_{j}<y_{j}, \text { for } j \neq i .
$$

Clearly, (9) implies (8). We have:

Theorem 14 Let $N=2$ and let conditions (4), (6) and (9) hold. Then $U$ is order preserving.

If $(H 1)-(H 2)$ hold, then $U$ also satisfies (H3) and the statement of Theorem 6 is valid.

Proof. Condition (8) gives us that, when two solutions with the same initial data become different, they do that simultaneously in all directions. Indeed, suppose that, for the two solutions $x(\cdot), \tilde{x}(\cdot)$, and $t_{0} \in[0, T]$, we have that $x_{i}\left(t_{0}\right)=\tilde{x}_{i}\left(t_{0}\right)$, for $i=1,2$, but $x_{1}(t)>\tilde{x}_{1}(t)$ and $x_{2}(t)=\tilde{x}_{2}(t)$ for $t \in\left(t_{0}, t_{0}+\delta\right)$. Then,

$$
\frac{d}{d t}\left(x_{2}(t)-\tilde{x}_{2}(t)\right)=f_{2}(x(t))-f_{2}(\tilde{x}(t))>0, \text { for } t \in\left(t_{0}, t_{0}+\delta\right),
$$

so that $x_{2}(t)>\tilde{x}_{2}(t)$, which is a contradiction.

The stronger condition (9) implies that it is not possible that

$$
\begin{aligned}
x_{i}\left(t_{0}\right) & =\tilde{x}_{i}\left(t_{0}\right), \text { for } i=1,2, \\
x_{1}(t) & >\tilde{x}_{1}(t), x_{2}(t)<\tilde{x}_{2}(t), \text { for } t \in\left(t_{0}, t_{0}+\delta\right),
\end{aligned}
$$

since we then obtain

$$
\frac{d}{d t}\left(x_{2}(t)-\tilde{x}_{2}(t)\right)=f_{2}(x(t))-f_{2}(\tilde{x}(t))>0, \text { for } t \in\left(t_{0}, t_{0}+\delta\right),
$$


which is a contradiction.

Using again (9) or (8) one can see that after $t_{0}$ either the two components $x_{i}(t)$ remain different for all $t$, or for some $t_{1}>t_{0}$ we have again $x\left(t_{1}\right)=\widetilde{x}\left(t_{1}\right)$. Indeed, if $x_{1}\left(t_{1}\right)=\widetilde{x}_{1}\left(t_{1}\right), x_{2}\left(t_{1}\right)>\widetilde{x}_{2}\left(t_{1}\right)$ and $x_{i}(t)>\widetilde{x}_{i}(t)$, for $t \in\left[t_{1}-\delta, t_{1}\right), i=1,2$ (for instance), then

$$
\frac{d}{d t}\left(x_{1}(t)-\tilde{x}_{1}(t)\right)=f_{1}(x(t))-f_{1}(\tilde{x}(t))>0, \text { for } t \in\left[t_{1}-\delta, t_{1}\right),
$$

so that $x_{1}\left(t_{1}\right)>\widetilde{x}_{1}\left(t_{1}\right)$, a contradiction. We repeat again the argument in the following interval and conclude that for any given moment of time $t$ the set $U\left(t, x_{0}\right)$ is totally ordered.

Moreover, as this set is compact, then we have the existence of a maximal and minimal elements in $U\left(t, x_{0}\right)$. We conclude by applying Theorem 13 .

Remark 15 We have proved here the additional property that the set $U(t, x)$ is totally ordered. This is valid also for the map $G$ if we assume condition (9).

It is also clear that in the case of the map $U$ it is sufficient to assume (9) for $x, y \in \mathbb{R}_{+}^{2}$.

\subsubsection{A biochemical control circuit}

As an application of the previous results we consider a model on the control of protein synthesis in the cell. Consider the two dimensional system (see Arnold and Chueshov [1], Chueshov [10] for the random case and Smith [20] for the deterministic case):

$$
\left\{\begin{array}{l}
x_{1}^{\prime}(t)=g\left(x_{2}(t)\right)-\alpha_{1} x_{1}(t) \\
x_{2}^{\prime}(t)=x_{1}(t)-\alpha_{2} x_{2}(t) \\
x_{i}(0)=x_{0}^{i}
\end{array}\right.
$$

where $g$ satisfies $g(0) \geq 0$ and is a continuous strictly increasing sub-linear function, so that there exist $a, b>0$ such that

$$
|g(x)| \leq a+b|x|
$$

and

$$
\begin{aligned}
\alpha_{i} & >0, i=1,2 \\
\alpha_{1} \alpha_{2} & >\frac{(b+1)^{2}}{4} \text { or }\left\{\alpha_{1}>1 \text { and } \alpha_{2}>b\right\} .
\end{aligned}
$$

The unknowns represent concentrations of macromolecules in a cell, so they are supposed to be non-negative (see Smith [20] or Chueshov [10]). It is clear that (4) and (6) hold, which means that the SMSF $U$ is well defined.

This system satisfies $(H 1)-(H 2)$, so that it is dissipative. Indeed, according to (11)-(13) we have to consider two cases:

Case I: $\alpha_{1} \alpha_{2}>\frac{(b+1)^{2}}{4}$. We obtain that

$$
\begin{aligned}
\frac{1}{2} \frac{d}{d t}\|x\|^{2}=f_{1}(x) x_{1}+f_{2}(x) x_{2} & =g\left(x_{2}\right) x_{1}-\alpha_{1} x_{1}^{2}+x_{1} x_{2}-\alpha_{2} x_{2}^{2} \\
& \leq\left(a+b x_{2}\right) x_{1}-\alpha_{1} x_{1}^{2}+x_{1} x_{2}-\alpha_{2} x_{2}^{2} \\
& \leq a x_{1}+\left((b+1)-2(1-\varepsilon) \sqrt{\alpha} \sqrt{\alpha_{2}}\right) x_{1} x_{2} \\
& -(1-\varepsilon)\left(\sqrt{\alpha_{1}} x_{1}-\sqrt{\alpha_{2}} x_{2}\right)^{2}-\varepsilon\left(\alpha_{1} x_{1}^{2}+\alpha_{2} x_{2}^{2}\right) \\
& \leq-\frac{\varepsilon}{2} \alpha_{1} x_{1}^{2}-\frac{\varepsilon}{2} \alpha_{2} x_{2}^{2}+c_{1} \\
& \leq-c_{2}\|x\|^{2}+c_{1}
\end{aligned}
$$

for some $c_{i}>0, i=1,2$. Hence by Gronwall's Lemma $\|x(t)\|^{2} \leq e^{-2 c_{2} t}\left\|x_{0}\right\|^{2}+\frac{c_{1}}{c_{2}}$, and then $(H 1)-(H 2)$ follow. 
Case II: $\alpha_{1}>1$ and $\alpha_{2}>b$. Summing up the two equations we have

$$
\begin{aligned}
\frac{1}{2} \frac{d}{d t}\left(x_{1}+x_{2}\right)^{2}= & \left(g\left(x_{2}(t)\right)+x_{1}(t)-\alpha_{1} x_{1}(t)-\alpha_{2} x_{2}(t)\right)\left(x_{1}+x_{2}\right) \\
& \leq\left(a+\left(1-\alpha_{1}\right) x_{1}+\left(b-\alpha_{2}\right) x_{2}\right)\left(x_{1}+x_{2}\right) \\
& \leq c_{1}-c_{2}\left(x_{1}+x_{2}\right)^{2},
\end{aligned}
$$

for some $c_{i}>0$. Again by the Gronwall lemma we obtain that $(H 1)-(H 2)$ hold. The set $B_{0}$ is defined by $B_{0}=\left\{x \in \mathbb{R}_{+}^{2}: 0 \leq x_{1}+x_{2} \leq \frac{c_{1}}{c_{2}}+\delta, \delta>0\right\}$.

Therefore, (4) and $(H 1)-(H 2)$ hold. On the other hand, (9) is satisfied, and then Theorem 14 implies that the statement of Theorem 6 is valid.

If we assume, moreover, that $g$ is Lipschitz in a neighborhood of the fixed point $x_{*}\left(\right.$ resp. $\left.y^{*}\right)$, then the solution corresponding to this initial condition is unique, so that $x_{*}$ (resp. $y^{*}$ ) is globally attracting from below (resp. above).

If the function $g$ is uniformly bounded by a constant $a$ (as in Smith [20, p.58]) we can withdraw condition (13). Indeed, in that case we can use the variation of constants formula with the matrix $A=\left(\begin{array}{cc}-\alpha_{1} & 0 \\ 1 & -\alpha_{2}\end{array}\right)$ :

$$
x(t)=e^{A t} x_{0}+\int_{0}^{t} e^{A(t-s)}\left(\begin{array}{c}
g\left(x_{2}\right) \\
0
\end{array}\right) d s
$$

and properties $(H 1)-(H 2)$ are fulfilled since $|g(u)| \leq a$ and $\alpha_{i}>0$. Hence, the statement of Theorem 6 is valid.

Also, if $g(u)=g_{0}(u)+b u$, where $\left|g_{0}(u)\right| \leq a$, we can assume that $\alpha_{1} \alpha_{2}>b($ instead of (13)). In such a case the eigenvalues of the matrix $A=\left(\begin{array}{cc}-\alpha_{1} & b \\ 1 & -\alpha_{2}\end{array}\right)$ have negative real parts, and then using again (14), but putting $g_{0}$ instead of $g$, we obtain the desired result.

Remark 16 We also note that in this equation the set of fixed points is totally ordered (see Smith [20, p.58]).

\subsubsection{A biochemical control circuit in $\mathbb{R}^{N}$}

In the $N$-dimensional case consider the system

$$
\left\{\begin{array}{l}
x_{1}^{\prime}(t)=f_{1}\left(x_{N}, x_{1}\right) \\
x_{2}^{\prime}(t)=f_{2}\left(x_{1}, x_{2}\right) \\
\vdots \\
x_{N}^{\prime}=f_{N}\left(x_{N-1}, x_{N}\right) \\
x_{i}(0)=x_{0}^{i}
\end{array}\right.
$$

with the monotonicity condition

$$
f_{i}(x, \widetilde{x})<f_{i}(y, \widetilde{y}), \quad \text { if } \widetilde{x} \geq \widetilde{y} \text {, and } x<y, \forall i,
$$

which is the analogue of (9). Assume again that (4) holds.

We shall check that $U$ is order preserving.

Theorem 17 Let conditions (4), (6) and (16) hold. Then $U$ is order preserving. If $(H 1)-(H 2)$ hold, then $U$ satisfies also $(H 3)$ and the statement of Theorem 6 is valid. 
Proof. Suppose first that $U\left(T, x_{0}\right)$ has a maximal element $\bar{x} \in U\left(T, x_{0}\right)$ and let $\bar{x}(t)$ be the corresponding solution. Let $y_{0}<x_{0}$ and $y(\cdot) \in D\left(y_{0}, T\right)$ be such that $y(T) \not \leq \bar{x}$. As before, in such a case there cannot exist $t_{0}$ such that $y\left(t_{0}\right)=\bar{x}\left(t_{0}\right)$ by the concatenation argument, which leads to a contradiction. Then, there exist $t_{0} \in[0, T), \delta>0$ and $i, j$ such that

$$
\begin{gathered}
\bar{x}_{i}\left(t_{0}\right)=y_{i}\left(t_{0}\right), \bar{x}_{j}\left(t_{0}\right)>y_{j}\left(t_{0}\right), \bar{x}\left(t_{0}\right) \geq y\left(t_{0}\right), \\
\bar{x}_{i}(t)<y_{i}(t), \quad \text { for } t \in\left(t_{0}, t_{0}+\delta\right) .
\end{gathered}
$$

Thus, we also get that $\bar{x}_{j+1}(t)>y_{j+1}(t)$ for all $t \in\left(t_{0}, t_{0}+\delta_{2}\right)$, for some $\delta_{2}>0$. This is clear if $\bar{x}_{j+1}\left(t_{0}\right)>$ $y_{j+1}\left(t_{0}\right)$, and, if $\bar{x}_{j+1}\left(t_{0}\right)=y_{j+1}\left(t_{0}\right)$, then (16) implies that

$$
f_{j+1}\left(\bar{x}_{j}(t), \bar{x}_{j+1}(t)\right)>f_{j+1}\left(y_{j}(t), y_{j+1}(t)\right),
$$

for all $t \in\left(t_{0}, t_{0}+\delta_{2}\right)$, from which we can conclude that $\left(\bar{x}_{j+1}(t)-y_{j+1}(t)\right)^{\prime}>0$ and so $\bar{x}_{j+1}(t)>y_{j+1}(t)$. Further, $\bar{x}_{j+2}(t)>y_{j+2}(t)$ for all $t \in\left(t_{0}, t_{0}+\delta_{3}\right)$, for some $\delta_{3}>0$, since, otherwise, $\bar{x}_{j+2}\left(t_{0}\right)=y_{j+2}\left(t_{0}\right)$, $\bar{x}_{j+2}(t) \leq y_{j+2}(t)$, for $t \in\left[t_{0}, t_{0}+\delta_{4}\right]$, and condition (16) leads again to a contradiction. In a similar way, by repeating the argument for $j+3, j+4, \ldots$, up to $j+k=i$, we get a contradiction.

Since a similar argument is also valid for the minimal element, it follows that $U$ is order preserving.

Let us now prove that we can obtain maximal and minimal elements in $U\left(T, x_{0}\right)$. First, if there exist $t_{0} \in[0, T], x(\cdot), y(\cdot) \in D\left(x_{0}, T\right)$ such that $x(t) \neq y(t)$, for $t \in\left(t_{0}, t_{0}+\delta\right)$, and $x(t)=y(t)$, for $t \leq t_{0}$, then all of their coordinates must be different. Indeed, if not, there exist $x_{i}, y_{i}, x_{j}, y_{j}$ such that

$$
\begin{aligned}
x_{i}\left(t_{0}\right) & =y_{i}\left(t_{0}\right), x_{i}(t)<y_{i}(t), \text { for } t \in\left(t_{0}, t_{0}+\delta\right), \\
x_{j}(t) & =y_{j}(t), \text { for } t \in\left[t_{0}, t_{0}+\delta\right) .
\end{aligned}
$$

Then, $x_{i+1}(t)<y_{i+1}(t)$, for $t \in\left(t_{0}, t_{0}+\widetilde{\delta}\right)$ and some $\widetilde{\delta}<\delta$, since, otherwise, by (16) we have $\frac{d}{d t}\left(x_{i+1}(t)-y_{i+1}(t)\right)<$ 0 , a contradiction. But the same is true for $i+2, i+3, \ldots, i+k=j$, from which we get a contradiction, because $x_{j}(t)=y_{j}(t)$, for $t \in\left[t_{0}, t_{0}+\delta\right)$. Moreover, the same argument leads us to prove that it is not possible to have

$$
\begin{aligned}
& x\left(t_{0}\right)=y\left(t_{0}\right), \\
& x_{i}(t)>y_{i}(t), x_{j}(t)<y_{j}(t), \text { for } t \in\left(t_{0}, t_{0}+\delta\right) .
\end{aligned}
$$

Then, in some $\left(t_{0}, t_{0}+\widehat{\delta}\right)$ we have $x_{j}(t)>y_{j}(t)$ for all $j$. After the time $t_{0}$ there can exist another moment $t_{1}>t_{0}$ where one of the components, say $i$, satisfies again $x_{i}\left(t_{1}\right)=y_{i}\left(t_{1}\right)$ and change the inequality, i.e. $x_{i}(t)<y_{i}(t)$ in $\left(t_{1}, t_{1}+\delta\right)$. Since this is the first time when this occurs, we have $x_{j}(t) \geq y_{j}(t)$, for $t \in\left[t_{0}, t_{1}\right]$ and all $j$. We claim now that $x_{j}\left(t_{1}\right)=y_{j}\left(t_{1}\right)$ for all $j$. By contradiction, suppose that there is $j$ such that $x_{j}\left(t_{1}\right)>y_{j}\left(t_{1}\right)$. Hence, either $x_{j+1}\left(t_{1}\right)>y_{j+1}\left(t_{1}\right)$ or $x_{j+1}\left(t_{1}\right)=y_{j+1}\left(t_{1}\right)$. In the last case we have by (16)

$$
\frac{d}{d t}\left(x_{j+1}-y_{j+1}\right)=f_{j+1}\left(x_{j}(t), x_{j+1}(t)\right)-f_{j+1}\left(y_{j}(t), y_{j+1}(t)\right)>0, \text { for } t \in\left(t_{1}, t_{1}+\delta_{1}\right),
$$

from which $x_{j+1}(t)>y_{j+1}(t)$, for $t \in\left(t_{1}, t_{1}+\delta_{1}\right)$, in both cases. Further, for $j+2$ we have again that either $x_{j+2}\left(t_{1}\right)>y_{j+2}\left(t_{1}\right)$ or $x_{j+2}\left(t_{1}\right)=y_{j+2}\left(t_{1}\right)$. In both cases it holds again that $x_{j+2}(t)>y_{j+2}(t)$, for $t \in\left(t_{1}, t_{1}+\delta_{2}\right)$. For the second case if $x_{j+2}(t) \leq y_{j+2}(t)$, then applying (16) again we have

$$
\frac{d}{d t}\left(x_{j+2}-y_{j+2}\right)=f_{j+2}\left(x_{j+1}(t), x_{j+2}(t)\right)-f_{j+2}\left(y_{j+1}(t), y_{j+2}(t)\right)>0, \text { for } t \in\left(t_{1}, t_{1}+\delta_{2}\right),
$$

a contradiction. We continue the same argument for $j+3, j+4$, etc. When $j+k=i$ we obtain a new contradiction, so that $x_{j}\left(t_{1}\right)=y_{j}\left(t_{1}\right)$ for all $j$. But then arguing as before in some $\left(t_{1}, t_{1}+\delta_{3}\right)$ we have $x_{j}(t)<y_{j}(t)$ for all $j$. 
This argument implies that $U\left(T, x_{0}\right)$ is totally ordered for any $\left(T, x_{0}\right)$. Hence, a maximal and a minimal elements exist and then $U$ is order preserving.

We have already proved in Theorem 13 that $(H 1)-(H 2)$ imply $(H 3)$.

We now consider the $N$-dimensional variant of the previous model on a biochemical control circuit:

$$
\left\{\begin{array}{l}
x_{1}^{\prime}(t)=g\left(x_{N}(t)\right)-\alpha_{1} x_{1}(t) \\
x_{2}^{\prime}(t)=x_{1}(t)-\alpha_{2} x_{2}(t) \\
\vdots \\
x_{N}^{\prime}(t)=x_{N-1}(t)-\alpha_{N} x_{N}(t) \\
x_{i}(0)=x_{0}^{i}
\end{array}\right.
$$

where $\alpha_{i}>0$ and

$$
\begin{gathered}
\alpha_{i} \alpha_{i+1}>1, i=2, \ldots, N-1, \alpha_{N} \alpha_{1}>b^{2}, \text { or } \\
\alpha_{i}>1, i=1, \ldots, N-1, \alpha_{N}>b .
\end{gathered}
$$

Here, $g(0) \geq 0$ and is a continuous strictly increasing function such that $|g(x)| \leq a+b|x|$. The variables $x_{i}$ take only non-negative values.

Since (4), (6) and (16) are satisfied, the associated multi-valued semi-flow $U\left(t, x_{0}\right)$ is order preserving. On the other hand, (18) implies that this system satisfies $(H 1)-(H 2)$. Indeed, consider again two cases:

Case I: $\alpha_{i} \alpha_{i+1}>1, i=2, \ldots, N-1, \alpha_{N} \alpha_{1}>b^{2}$. We get

$$
\begin{aligned}
& \frac{1}{2} \frac{d}{d t}\|x\|^{2}=\sum_{i=1}^{N} f_{i}(x) x_{i}=g\left(x_{N}\right) x_{1}-\sum_{i=1}^{N} \alpha_{i} x_{i}^{2}+\sum_{i=1}^{N-1} x_{i} x_{i+1} \\
& \leq\left(a+b x_{N}\right) x_{1}-\frac{1}{2}\left(\alpha_{1} x_{1}^{2}+\alpha_{N} x_{N}^{2}\right) \\
& +\sum_{i=1}^{N-1}\left(x_{i} x_{i+1}-\frac{1}{2}\left(\alpha_{i} x_{i}^{2}+\alpha_{i+1} x_{i+1}^{2}\right)\right) \\
& \leq a x_{1}+(b-(1-2 \varepsilon)) \sqrt{\alpha_{1}} \sqrt{\alpha_{N}} x_{1} x_{N} \\
& -\left(\frac{1}{2}-\varepsilon\right)\left(\sqrt{\alpha}_{1} x_{1}-\sqrt{\alpha_{2} x_{N}}\right)^{2}-\varepsilon\left(\alpha_{1} x_{1}^{2}+\alpha_{2} x_{N}^{2}\right) \\
& +\sum_{i=1}^{N-1}\left(\left(1-(1-2 \varepsilon) \sqrt{\alpha_{i}} \sqrt{\alpha_{i+1}}\right) x_{i} x_{i+1}\right. \\
& \left.-\left(\frac{1}{2}-\varepsilon\right)\left(\sqrt{\alpha_{i}} x_{i}-\sqrt{\alpha_{i+1}} x_{i+1}\right)^{2}-\varepsilon\left(\alpha_{i} x_{i}^{2}+\alpha_{i+1} x_{i+1}^{2}\right)\right) \\
& \leq c_{1}-c_{2}\|x\|^{2} \text {, }
\end{aligned}
$$

for $c_{i}>0, i=1,2$, so that by Gronwall's lemma $(H 1)-(H 2)$ hold.

Case II: $\alpha_{i}>1, i=1, \ldots, N-1, \alpha_{N}>b$. The proof is similar to that of the two dimensional case.

Hence, Theorem 17 implies that the statement of Theorem 6 is valid. If we assume, moreover, that $g$ is Lipschitz in a neighborhood of the fixed points $x_{*}$ (resp. $\left.y^{*}\right)$, then the solutions corresponding to these initial conditions are unique, so that $x_{*}$ (resp. $\left.y^{*}\right)$ is globally attracting from below (resp. above).

As in the two dimensional case if the function $g$ is uniformly bounded by a constant $a$ we can withdraw condition (18) and use for the proof the variation of constants formula.

Also, if $g(u)=g_{0}(u)+b u$, where $\left|g_{0}(u)\right| \leq a$, we can assume that $\alpha_{1} \alpha_{2} \cdots \alpha_{N}>b$ (instead of (18)). In such a case the eigenvalues of the matrix $A$, in which the only non-zero elements are $a_{i i}=-\alpha_{i}, a_{i+1, i}=1$, $a_{1 N}=b$, have negative real parts, and then using again (14), but putting $g_{0}$ instead of $g$, we obtain the desired result.

\subsection{Delay differential equations: the scalar case}

Let $h>0$ be a given positive number (the delay time) and denote by $\mathcal{C}$ the Banach space $C([-h, 0] ; \mathbb{R})$ endowed with the norm $\|\psi\|=\sup _{\sigma \in[-h, 0]}|\psi(\sigma)|$. However, it is sometimes useful to consider the solutions as mappings from $\mathbb{R}$ into $\mathbb{R}$. If $x \in C([-h,+\infty), \mathbb{R})$ for any $t \geq 0$ we denote by $x_{t}$ the element in $\mathcal{C}$ given by $x_{t}(s)=x(t+s)$ for all $s \in[-h, 0]$. We consider the usual partial order in $C([-h, 0], \mathbb{R})$, i.e

$$
x \leq y \Leftrightarrow x(\theta) \leq y(\theta), \text { for all } \theta \in[-h, 0] .
$$


We say that $x<y$ if $x \leq y$ and there exists $\theta \in[-h, 0]$ such that $x(\theta)<y(\theta)$.

We will now consider the functional differential equation with finite delay:

$$
x^{\prime}(t)=f\left(x_{t}\right), \quad x_{0}=\psi \in \mathcal{C},
$$

where $f: \mathcal{C} \rightarrow \mathcal{C}$. We assume that for any initial data $\psi \in \mathcal{C}$ there exists at least one solution $x(\cdot)$ and also that each solution is defined globally in time. This allows us to define an associated multi-valued semi-flow. As before denote by $S\left(x_{0}\right) \subset C([0,+\infty), \mathcal{C})$ the set of all solutions corresponding to the initial condition $x_{0}$ and by $S\left(x_{0}, T\right) \subset C([0, T], \mathcal{C})$ the set

$$
S\left(x_{0}, T\right)=\left\{x(\cdot) \in C([0, T], \mathcal{C}): x(\cdot) \text { is a solution of }(19) \text { and } x(0)=x_{0}\right\} .
$$

As in the previous cases, it is usual to consider non-negative solutions. Let $X=C\left([-h, 0], \mathbb{R}_{+}\right.$) (in general we can consider the phase space $X=C([-h, 0], Z)$, where $Z$ is a closed subset of $\mathbb{R})$ and assume that at least one globally defined solution with $x(t) \in X$, for all $t \geq 0$, exists for any initial data in $X$. Denote by $U\left(x_{0}\right) \subset C([0,+\infty), X)$ the set of all solutions with initial condition $x_{0}$ such that $x(t) \geq 0$ for all $t \geq 0$. Define also the set

$$
D\left(x_{0}, T\right)=\left\{x(\cdot) \in C([0, T], \mathcal{C}): x(\cdot) \text { is a solution of }(19) \text { and } x(0)=x_{0}, x(t) \geq 0\right\} .
$$

Then the maps $G: \mathbb{R}_{+} \times \mathcal{C} \longrightarrow P(\mathcal{C}), U: \mathbb{R}_{+} \times X \longrightarrow P(X):$

$$
\begin{aligned}
& G(t, x)=\left\{x_{t}: x(\cdot) \in S\left(x_{0}\right)\right\}, \\
& U(t, x)=\left\{x_{t}: x(\cdot) \in U\left(x_{0}\right)\right\},
\end{aligned}
$$

are SMSF (see [9]).

As in the case of ordinary differential equations we can easily prove that the fixed points coincide with the set of constant functions $\psi(\theta) \equiv x \in \mathbb{R}$, such that $f(\psi)=0$.

Some general conditions providing the existence of globally defined solutions and also $(H 1)-(H 3)$ (and hence the existence of a global compact attractor) are given in [9]. We will not write them here and, instead, we consider the following particular case (which contains for instance models of populations with variable age of maturation [7]):

$$
x^{\prime}(t)=f\left(x_{t}\right)=F_{0}(x(t))+F_{1}(x(t-h))+\int_{-h}^{0} b(s, x(t+s)) d s,
$$

where we assume that $F_{i} \in C(\mathbb{R}, \mathbb{R}), i=0,1, b \in C([-h, 0] \times \mathbb{R}, \mathbb{R})$ and the following conditions:

(B) There exist scalar positive functions $m_{i} \in L^{1}([-h, 0]), i=0,1$, such that

$$
|b(s, x)| \leq m_{0}(s)+m_{1}(s)|x| \text {, for all }(s, x) .
$$

(F) There exist positive constants $k_{1}, k_{2}, \alpha$ and $\beta$ such that

$$
\begin{gathered}
x F_{0}(x) \leq-\alpha x^{2}+\beta, \\
\left|F_{1}(x)\right|^{2} \leq k_{1}^{2}+k_{2}^{2}|x|^{2}, \text { for all } x .
\end{gathered}
$$

Denote $M_{i}=\int_{-h}^{0} m_{i}(s) d s$. Assume also that the following inequalities hold:

$$
\begin{aligned}
2 M_{1} e h & <1, \\
k_{2}^{2} & <e^{-1} \alpha\left(\alpha-\lambda^{*}\right), \\
\lambda^{*} & <\alpha,
\end{aligned}
$$

where $\lambda^{*} \in\left(\lambda_{0}, \lambda_{1}\right)$ and $\lambda_{i}$ are the two solutions of the equation $\lambda e^{-\lambda h}=2 M_{1}$ if $M_{1}>0\left(\lambda^{*}=0\right.$ if $\left.M_{1}=0\right)$.

Then all the solutions are globally defined in time and we can define the strict multi-valued semi-flow $G$ [9]. Let, moreover, one of the following conditions hold: 
1. For all $\psi \in C\left([-h, 0], \mathbb{R}_{+}\right)$with $\psi(0)=0$ we have

$$
F_{0}(\psi(0))+F_{1}(\psi(-h))+\int_{-h}^{0} b(s, \psi(s)) d s=0 ;
$$

2. For all $\psi \in C([-h, 0], \mathbb{R})$ with $\psi(0)<0$ we have

$$
F_{0}(\psi(0))+F_{1}(\psi(-h))+\int_{-h}^{0} b(s, \psi(s)) d s>0 .
$$

Then for any initial data in $X$ there exists at least one globally defined solution such that $x(t) \geq 0$, for any $t \geq 0$ [9]. Hence, we can define also the SMSF $U$. It is also proved in [9] that $G$ and $U$ have a global compact invariant attractor.

Let us study now whether the SMSF $G$ (resp. $U$ ) is order preserving.

Theorem 18 We assume that

$$
\begin{aligned}
f\left(\psi_{1}\right) & <f\left(\psi_{2}\right), \text { for any } \psi_{i} \in C([-h, 0] ; \mathbb{R}) \\
\text { such that } \psi_{1}(0) & =\psi_{2}(0) \text { and } \psi_{1}<\psi_{2} .
\end{aligned}
$$

Let $(H 1)-(H 3)$ hold and let $f$ be a continuous and bounded map. Then $G$ is order preserving and the statement of Theorem 6 is valid. If either (25) or (26) holds and (27) is satisfied for $\psi_{i} \in C\left([-h, 0], \mathbb{R}_{+}\right)$, then the same is valid for $U$.

Proof. We shall write the proof for the SMSF $G$. For $U$ the proof is exactly the same. First let us prove that $G\left(t, x_{0}\right)$ has a maximal and a minimal element. Let

$$
A_{t}(0)=\left\{x_{t}(0): x_{t} \in G\left(t, x_{0}\right)\right\}
$$

and denote by $M_{t}, m_{t}$ the maximal and minimal elements of $A_{t}(0)$, respectively. We shall prove the existence of a solution $\bar{x}(\cdot) \in S\left(x_{0}, t\right)$ such that

$$
\bar{x}(\tau) \geq x(\tau), \text { for all } x(\cdot) \in S\left(x_{0}, t\right), \tau \in[0, t] .
$$

For this purpose we define the following sequence of solutions with initial condition $x_{0}$ :

$$
\begin{aligned}
x^{1}(\cdot) \text { such that } x^{1}(t) & =M_{t}, \\
x^{2}(\cdot) \text { such that }: x^{2}\left(\frac{t}{2}\right) & =M_{\frac{t}{2}}, x^{2}(t)=M_{t}, \\
x^{4}(\cdot) \text { such that } x^{4}\left(\frac{k t}{4}\right) & =M_{\frac{k t}{4}}, k=1, \ldots, 4, \\
& \vdots \\
x^{2^{n}}(\cdot) \text { such that } x^{2^{n}}\left(\frac{k t}{2^{n}}\right) & =M_{\frac{k t}{2^{n}}}, \text { for } k=1,2, \ldots, 2^{n},
\end{aligned}
$$

which exists because the concatenation of solutions is a solution as well. In view of $(H 2)$ and the boundednes of $f$ this sequence and its derivative $\frac{d}{d t} x^{2^{n}}$ are bounded in $C([0, t], \mathbb{R})$, so that the Ascoli-Arzelà theorem implies that $x^{2^{n}}$ is pre-compact in $C([0, t], \mathbb{R})$. The continuity and boundedness of $f$ allows us to apply Lebesgue theorem and pass to the limit in the integral expression

$$
x^{2^{n}}(\tau)=x_{0}(0)+\int_{0}^{\tau} f\left(x_{s}^{2^{n}}\right) d s,
$$


so that the limit function $\bar{x}(\cdot)$ is also a solution. We note that $\bar{x}(\tau)=M_{\tau}$, for all $\tau \in A$, where $A$ is a dense subset of $[0, t]$. By continuity $\bar{x}(\tau)=M_{\tau}$, for all $\tau \in[0, t]$. Hence, $\bar{x}(\tau) \geq x(\tau)$, for all $x(\cdot) \in S\left(x_{0}, t\right)$, $\tau \in[0, t]$, and then $\bar{x}_{t} \geq x_{t}$, for any $x_{t} \in G\left(t, x_{0}\right) . \bar{x}_{t}$ is the desired maximal element of $G\left(t, x_{0}\right)$.

In a similar way we obtain the existence of a minimal element $\underline{x}_{t}$.

Let now $y_{0}<x_{0}$. We shall prove that

$$
y(\tau) \leq \bar{x}(\tau), \text { for all } \tau \in[0, t], y(\cdot) \in S\left(y_{0}, t\right),
$$

where $\bar{x}(\cdot)$ is the maximal solution corresponding to $x_{0}$. If this is not the case, then there exists $t_{0}<t$ such that

$$
\begin{aligned}
y\left(t_{0}\right) & =\bar{x}\left(t_{0}\right) \\
y(\tau) & \leq \bar{x}(\tau), \text { for any } \tau \leq t, \\
y(\tau) & >\bar{x}(\tau), \text { for any } \tau \in\left(t_{0}, t_{0}+\delta\right) .
\end{aligned}
$$

We have now two cases.

Case I: There exists $\tau \in\left[t_{0}-h, t_{0}\right)$ such that $y(\tau)<\bar{x}(\tau)$. In this case condition (27) and the continuity of $f$ imply that $\frac{d}{d \tau}(\bar{x}-y)>0$, in $\left[t_{0}, t_{0}+\widetilde{\delta}\right)$, which leads to a contradiction.

Case II: $\bar{x}(\tau)=y(\tau)$, for all $\tau \in\left[t_{0}-h, t_{0}\right]$. In such a case we can define the solution

$$
z(\tau)=\left\{\begin{array}{c}
\bar{x}(\tau), \text { if } \tau \in\left[-h, t_{0}\right], \\
y(\tau), \text { if } \tau \in\left[t_{0}, t\right]
\end{array}\right.
$$

which belongs to $S\left(x_{0}, t\right)$. This contradicts that $\bar{x}(\cdot)$ is a maximal solution for $x_{0}$.

In a similar way we consider the case $y_{0}>x_{0}$. We have proved that $G$ (resp. $U$ ) is order preserving. We conclude the proof by applying Theorem 6 .

Remark 19 In the case of the map $G$ it could be possible to prove this result under less restrictive conditions, as happens for ordinary differential equations (see Theorem 10). However, in applications, the solutions usually take non-negative values, so that we omit here such results.

Consider now equation (20).

Theorem 20 Let conditions (21)-(24) hold. Assume also that the function $b(s, x)$ is strictly increasing with respect to $x$ (s constant) and that $F_{1}$ is non-decreasing. Then $G$ is order preserving and the statement of Theorem 6 is valid. If one of conditions (25)-(26) holds, then the same is true for U.

Proof. Conditions $(H 1)-(H 3)$ are proved in Caraballo et. al. [9]. We note also that the continuity of the functions $F_{i}, b$ implies that $f$ is continuous and bounded. Further, consider $\psi_{i}$ such that $\psi_{1}(0)=\psi_{2}(0)$ and $\psi_{1}<\psi_{2}$. Then

$$
f\left(\psi_{1}\right)-f\left(\psi_{2}\right)=F_{1}\left(\psi_{1}(-h)\right)-F_{1}\left(\psi_{2}(-h)\right)+\int_{-h}^{0}\left(b\left(s, \psi_{1}(s)\right)-b\left(s, \psi_{2}(s)\right)\right) d \tau<0 .
$$

Therefore, (27) is satisfied. Hence, we can apply Theorem 18.

Acknowledgments. We would like to express our thanks to the referees for helpful and interesting comments and suggestions.

This work was partially supported by the MCYT (Ministerio de Ciencia y Tecnología, Spain) and FEDER (Fondo Europeo de Desarrollo Regional) grants BFM2002-03512 and BFM2002-03068, and by the Consejería de Cultura y Educación (Comunidad Autónoma de Murcia) grant PI-8/00807/FS/01. 


\section{References}

[1] Arnold L. and Chueshov I.D., Order-preserving random dynamical systems: equilibria, attractors, applications. Dynam. Stability Systems 13 (1998), no. 3, 265-280.

[2] Arrieta J.M., Rodríguez-Bernal A. and Vidal-López A., Regiones atractoras delimitadas por puntos de equilibrio extremales para ecuaciones de reacción-difusión, Proceedings in CDRom of the XVIII CEDYA-VIII CMA, Tarragona (Spain), 2003.

[3] Aubin J.P. and Frankowska H., Set-Valued Analysis, Birkhäuser, Boston, 1990.

[4] Babin A.V., Attractors of the generalized semigroup generated by an elliptic equation in a cylindrical domain, Russian Acad. Sci. Izv. Math., 44 (1995), 207-223.

[5] Ball J.M., On the asymptotic behavior of generalized processes with applications to nonlinear evolution equations, J. Differential Equations, (27) 1978, 224-265.

[6] Ball J.M., Continuity properties and global attractors of generalized semiflows and the Navier-Stokes equations, in "Mechanics: from theory to computation", Springer, New York, 2000, pp.447-474.

[7] Brauer F. and Castillo-Chávez C., Mathematical Models in Population Biology and Epidemiology, Springer, New York, 2001.

[8] Caraballo T., Marín-Rubio P. and Robinson J.C., A comparison between two theories for multivalued semiflows and their asymptotic behaviour, Set-Valued Analysis, 11 (2003), 297-322.

[9] Caraballo T., Marín-Rubio P. and Valero J., Autonomous and non-autonomous attractors for differential equations with delays, J. Differential Equations, 208 (2005), 9-41.

[10] Chueshov I.D., Monotone random systems: theory and applications, Lecture Notes in Mathematics 1779, Springer-Verlag, Berlin, 2002.

[11] Elmounir O. and Simondon F., Attracteurs compacts pur des problèmes d'evolution sans unicité, Ann. Fac. Sci. Toulouse Math., Série 6, vol. IX, Fasc. 4 (2000), 631-654.

[12] Hartman Ph., Ordinary Differential Equations, Classics in Applied Mathematics 38, SIAM, 2002.

[13] Hirsch M., Positive equilibria and convergence in subhomogeneous monotone dynamics, in "Comparison methods and stability theory" (Waterloo, ON, 1993), Lecture Notes in Pure and Appl. Math. 162, Dekker, New York, 1994, pp.169-188.

[14] Kapustyan A.V., Melnik V.S. and Valero J., Attractors of multivalued dynamical processes generated by phase-field equations, Internat. J. Bifurcat. Chaos, 13 (2003), 1969-1983.

[15] Kapustyan A.V., Global attractors of a nonautonomous reaction-diffusion equation, Diff. Uravnenya, 38 (2002), 1378-1381 (English translation in Differential Equations, 38 (2002), 1467-1471).

[16] Mackey M.C. and Glass L., Oscillation and chaos in physiological control systems, Science 197 (1977), 287-289.

[17] Melnik V.S. and Valero J., On attractors of multivalued semi-flows and differential inclusions. Set-Valued Anal. 6 (1998), no. 1, 83-111

[18] Melnik V.S. and Valero J., On global attractors of multivalued semiprocesses and nonautonomous evolution inclusions, Set-Valued Anal., 8 (2000), 375-403.

[19] Roxin E., Stability in general control systems, J.Differential Equations, 1 (1965), 115-150.

[20] Smith H.L., Monotone dynamical systems. An introduction to the theory of competitive and cooperative systems, Mathematical Surveys and Monographs 41, American Mathematical Society, Providence, RI, 1995.

[21] Temam R., Infinite-Dimensional Dynamical Systems in Mechanics and Physics, Springer-Verlag, New York 1988 (2nd edition in 1996).

[22] Valero J., Attractors of parabolic equations without uniqueness, J. Dynamics Differential Equations, 13 (2001), 711-744. 